\title{
L'histoire sociale et la constitution de la norme linguistique - les modèles français, espagnol et portugais
}

\section{Dietmar Osthus (Université de Duisbourg-Essen, Allemagne)}

\author{
1. Introduction: les normes linguistiques et l'historiographie linguistique
}

La constitution des normes linguistiques - le sujet principal de nos débats - comporte plusieurs aspects. D'un côté il n'y a aucun doute que les normes linguistiques se conçoivent sous un aspect idéel. Le discours normatif - terme employé par Alain Berrendonner pour décrire l'ensemble des activités ayant pour but d'influencer les comportements linguistiques - et les évolutions de ce discours au cours de l'histoire sont soumis aux différents courants intellectuels comme aux idéologies dominantes. Les idées concrètes que les autorités en matières de langue se font de ce qui est le bon usage d'une langue donnée font partie de l'histoire des idées, telle qu'elle a été décrite dans des oeuvres comme ceux de Sylvain Auroux. Dans l'historiographie des normes linguistiques on observe justement la tendance à s'appuyer exclusivement sur les sources métalinguistiques, c'est-à-dire les grammaires, les dictionnaires et les oeuvres para-grammaticales. Par conséquent on ne connaît que trop bien les doctrines défendues par les différents auteurs des oeuvres normatifs. Les frictions à l'intérieur du groupe des grammairiens ou des lexicographes - pensons à titre d'exemple au conflit entre Furetière et l'Académie française - font partie du savoir partagé de tout historien de la langue.

De l'autre côté, limiter la constitution des normes linguistiques à la naissance des conceptions normatives signifierait de négliger la dimension sociale de toute norme linguistique ${ }^{1}$. L'histoire des normes linguistiques comprend à côté de l'évolution de l'idée d'une langue légitime (Bourdieu 1983) impérativement l'histoire de l'adhésion sociale aux normes prônées. Cependant cette histoire sociale de la normalisation est beaucoup moins connue. Répondre à plusieurs questions cruciales de l'histoire sociale de la normalisation s'avère nettement plus difficile que la reconstruction des idées sur la norme: Comment les tentatives de normalisation sont-elles reçues par les différents secteurs de la population? Ou, dans une perspective plus globale: Dans quelles circonstances socioéconomiques la normalisation de la langue a une chance de s'imposer? Quelles sont les conditions sociales qui favorisent les activités normalisantes?

L'exemple de l'histoire de l'alphabétisation - elle même un facteur important dans l'histoire de la normalisation linguistique - démontre l'importance de transgresser les bornes de l'histoire des institutions et de celle des idées. François Furet et Jacques Ozouf insistent ainsi sur l'appartenance de l'alphabétisation à l'histoire sociale;

1 Mattheier (1999:17s.) souligne l'urgent besoin d'intégrer la sociologie comme l'histoire sociale dans la description et l'interprétation de l'histoire linguistique. Pourtant le cadre sociologique de l'histoire des langues reste encore à élaborer: « Einmal wird deutlich, daß Sprachhistoriker offensichtlich weitgehend ohne klare Vorstellungen über die gesellschaftlichen Strukturen und Entwicklungen, die die Soziologie und die Sozialgeschichte entwickelt haben, an die Analyse von sprachhistorischen Vorgän gen gehen, die ohne ein soziologisches Grundgerüst nicht angemessen beschrieben werden können. Zugleich muß jedoch fest gestellt werden, daß ein derartiger soziologischer Rahmen für Sprachgeschichte noch nicht erarbeitet worden ist. Zwar führen die Ansatzpunkte, Sprache als soziales Handeln aufzufassen, sicherlich auf den richtigen Weg. Es wird jedoch in den kommen den Jahren eine der zentralen Aufgaben der historischen Soziolinguistik sein, hier gesicherte sozialwissenschaftliche und sozialhistorische Grundlagen zu bekommen.“ 
l'alphabétisation est considérée comme un processus largement indépendant des grand événements politiques ou institutionnels - y inclus la Révolution française:

«Quant à l'histoire de l'alphabétisation elle-même, qui est celle d'une mise en place et d'une évolution lentes, à forte inertie sociale, elle a été confondue avec l'histoire des idées qu'on a pu se faire de l'école et de son rôle; or celle-ce n'est évidemment pas dotée du même rythme, et n'obéit pas aux mêmes pesanteurs » (Furet/Ozouf 1977:489).

Le constat d'une certaine négligence des aspects sociaux de la part des linguistes ne signifie tout de même pas l'absence totale de cette dimension. Suite aux réflexions très influentes de Pierre Bourdieu (1983) maints linguistes ont souligné les différentes dimensions sociales des approches normalisatrices. Les idéologies socio-politiques et l'interrelation qui existe entre les protagonistes de la normalisation linguistique et leur appartenance à certaines classes sociales ont bien été thématisées par les historiens de la langue. Diogo/Silvestre (1996:X) voient p.ex. dans l'agenda vernacular - c'est-à dire le projet de réformer et de normaliser la langue légitime - le reflet des «movimentos de ascensão e descenso no interior de la cour et la ville, processo, aliás, cujo início Auerbach recenseara já na corte de Luís XIV ». Ils identifient - pour le cas du discours normatif sur le portugais aux XVIIIe et XIXe siècles - la normalisation de la langue à un instrument de domination sociale:

« A reinvidicação e normalização do vernáculo revela-se, porém, ao longo de todo este período, um projecto central na política cultural de grupos sociais em ascenção, a qual é facilitada pelo triunfo desse vernáculo que serve ao mesmo tempo como mecanismo de mais fácil controlo de outros grupos sociais e da ordem estabelecida » (1996:VIIIf.).

D'autres auteurs insistent moins sur la domination sociale d'autres groupes que sur le rôle que joue la langue légitime pour la propre progression sociale (Settekorn 1988:33). Le prestige social (Thielemann 2004) lié à telle ou telle unité linguistique reflète le capital symbolique (Bourdieu 1983:43-45). La normalisation demande la participation de ceux dont les usages linguistiques sont régularisés. Cette participation peut bien être active et consciente comme c'est le cas des 'correcteurs de fautes vulgaires' (Osthus 2006:1538f.; Spillner 2004). L'affirmation que «Ce sont les Gascons qui se censurent et se corrigent eux-mêmes dans cet ouvrage » que l'on trouve dans la préface des fameux Occitanismes corrigés de Desgrouais (ed. 1801:XVI) témoigne d'une telle participation active à la correction linguistique ${ }^{2}$. L'adaptation aux modèles d'une langue standard ou au moins la reconnaissance d'une variété linguistique choisie comme étant supérieure à d'autres peut bien aussi se dérouler sous forme inconsciente ${ }^{3}$. Comme les normes linguistiques sont des normes sociales, la constitution des normes linguistiques est un processus conditionné par des facteurs sociaux comme la structure de la société, la formation de groupes sociaux et économiques ou bien l'évolution d'un système de l'éducation, comme l'affirme également John B. Thompson dans sa préface aux oeuvres métalinguistiques de Pierre Bourdieu:

«La normalisation et l'inculcation subséquentes de la langue officielle, et sa légitimation en tant que langue officielle de l'État-nation, n'était pas seulement une affaire strictement politique: il s'agissait également d'un processus graduel qui dépendait de tout un ensemble d'autres facteurs, comme le développement du système d'enseignement et la formation d'un marché de travail unifié. L'élaboration de manuels de grammaire, de dictionnaires et d'un corpus de textes exemplifiant les usages corrects, n'est que la manifestation la plus visible de ce processus de normalisation progressive » (Thompson 2001 [1991]:15).

\section{Les facteurs sociaux de la normalisation linguistique}

Pour mettre l'histoire de la normalisation linguistique en relation avec ces facteurs sociaux il faut tout d'abord clarifier les critères de ce qui est l'histoire sociale des normes linguistiques. Comment mesurer l'impact de la normalisation linguistique sur une société, voire une communauté linguistique? Vu les difficultés pour l'historien de reconstruire des faits essentiels comme les différents taux d'alphabétisme, il paraît incongru de vouloir déterminer en chiffres l'ancrage social des efforts normalisateurs. Ce que l'on peut faire, par contre, c'est de quantifier au moins certaines activités normatives comme p.ex. la ferveur de publier des oeuvres métalinguistiques à vocation normative. Le chiffre de publications n'est certes pas un indice certain - l'histoire de la réception des traités métalinguistique ne se résume pas en purs chiffres de publications ni même du total d'exemplaires vendus -, mais il nous fournit tout de même une première impression.

2 Spillner (2004:119) évoque ainsi l'origine provinciale de la plupart des auteurs des traités ayant pour but de corriger les régio nalismes.

3 Selon Corbeil (1983:285) cette adaptation aux modèles linguistiques dominants fait partie de l'enculturation: «Par enculturation, l'individu apprend et intègre des modèles pour chacun de ses comportements, y compris ses comportements linguistiques, c'est-à-dire qu'il acquiert une certaine conception de la manière dont il faut se comporter au sein de son groupe ou des infra groupes auxquels il a affaire pour s'y intégrer ou s'en faire accepter ». 


\subsection{Quantification de l'impact des activités normatives}

Les chiffres proviennent des trois grandes historiographies grammaticales; pour le français du Chronologisches Verzeichnis französischer Grammatiken (Stengel 1890 [ed. Niederehe 1976]), pour l'espagnol de la Bibliografia cronológica de la lingüistica, la gramática y la lexicografía del español (Niederehe 1995; 1999; 2005) et pour le portugais de la Historiografia gramatical 1500-1920 (Cardoso 1994). Les chiffres absolus ne sont pas comparables, vu que les trois inventaires obéissent à des critères tout à fait différents. Stengel (1890) fait preuve d'une vision étroite de la grammaticographie, tandis que Niederehe (1995ff.) inclut dans sa bibliographie - qui jusqu'alors ne couvre que la période jusqu'à l'an 1800 - toute la gamme de publications métalinguistiques. Ceci explique les chiffres beaucoup plus élevés. Cardoso (1994) inventarise les publications sur la langue portugaise comme celles publiées au Portugal sur les langues étrangères. Les différents types de publications (grammaires, manuels scolaires, polémiques etc.) se trouvent dans des registres séparés. Nous nous concentrerons ici sur la grammaticographie portugaise dans un sens étroit. Notre statistique quantitative des activités métalinguistiques donne ainsi le résultat suivant:

\begin{tabular}{|c|c|c|c|c|c|c|}
\hline & $\begin{array}{r}\text { avant } \\
1500\end{array}$ & $1500-1520$ & $1521-1540$ & 1541-1560 & $1561-1580$ & $1581-1600$ \\
\hline France & 3 & 1 & 5 & 12 & 10 & 17 \\
\hline Espagne & 93 & 60 & 75 & 151 & 199 & 221 \\
\hline Portugal & 0 & 0 & 2 & 1 & 0 & 1 \\
\hline
\end{tabular}

\begin{tabular}{|l|r|r|r|r|r|}
\hline & $\mathbf{1 6 0 1 - 1 6 2 0}$ & $\mathbf{1 6 2 1 - 1 6 4 0}$ & $\mathbf{1 6 4 1 - 1 6 6 0}$ & $\mathbf{1 6 6 1 - 1 6 8 0}$ & $\mathbf{1 6 8 1 - 1 7 0 0}$ \\
\hline France & 23 & 29 & 32 & 57 & 58 \\
\hline Espagne & 290 & 267 & 211 & 182 & 145 \\
\hline Portugal & 3 & 0 & 0 & 0 & 1 \\
\hline
\end{tabular}

\begin{tabular}{|l|r|r|r|r|r|}
\hline & $\mathbf{1 7 0 1 - 1 7 2 0}$ & $\mathbf{1 7 2 1 - 1 7 4 0}$ & $\mathbf{1 7 4 1 - 1 7 6 0}$ & $\mathbf{1 7 6 1 - 1 7 8 0}$ & $\mathbf{1 7 8 1 - 1 8 0 0}$ \\
\hline France & 46 & 46 & 52 & 63 & 162 \\
\hline Espagne & 254 & 228 & 263 & 318 & 494 \\
\hline Portugal & 1 & 8 & 3 & 3 & 13 \\
\hline
\end{tabular}

\begin{tabular}{|l|r|r|r|r|r|}
\hline & $1801-1820$ & $\mathbf{1 8 2 1 - 1 8 4 0}$ & $\mathbf{1 8 4 1 - 1 8 6 0}$ & $\mathbf{1 8 6 1 - 1 8 8 0}$ & $\mathbf{1 8 8 1 - 1 9 0 0}$ \\
\hline France & 256 & 458 & 523 & 319 & 266 \\
\hline Portugal & 13 & 27 & 47 & 57 & 63 \\
\hline
\end{tabular}

On voit d'abord que les activités métalinguistiques dans les trois pays subissent à peu près les mêmes tendances. Il y a tout au long de la période du $16 \mathrm{e}$ au $19 \mathrm{e}$ siècle une montée permanente de l'output grammatical avec une apogée en Espagne au début du 17e siècle et un déclin relatif dans la deuxième moitié du $17 \mathrm{e}$ siècle, un déclin qui coïncide avec la fin du siglo de oro. Le Portugal se trouve, en ce qui concerne la «productivité métalinguistique » un peu en retard par rapport à la France et à l'Espagne, ce qui s'explique entre autres par la situation relativement précaire du portugais pendant la période de l'union ibérique (1580-1640) et par la crise du système d'enseignement à partir de la restauration (Carvalho 2003. La 'traduction' des chiffres absolus en taux de croissance donne ainsi le résultat suivant:

\begin{tabular}{|l|r|r|r|r|r|}
\hline \multicolumn{2}{|c|}{ croissance des activités métalinguistiques en \% } & & & \\
\hline & $\mathbf{1 5 0 0 / 4 0 - 1 6 0 0 / 4 0}$ & $\mathbf{1 6 0 0 / 4 0 - 1 7 0 0 / 4 0}$ & $\begin{array}{r}\mathbf{1 6 8 0} / \mathbf{1 7 0 0} \\
\mathbf{1 7 8 0} / \mathbf{1 8 0 0}\end{array}$ & $\mathbf{1 7 0 0 / 4 0 - 1 8 0 0 / 4 0}$ & $\begin{array}{r}\mathbf{1 7 8 0} / \mathbf{1 8 0 0} \\
\mathbf{1 8 8 0} / \mathbf{1 9 0 0}\end{array}$ \\
\hline France & 766,7 & 76,9 & 179,3 & 676,1 & 64,2 \\
\hline Espagne & 312,6 & $-13,5 \%$ & 240,7 & & \\
\hline Portugal & 50 & 200 & 1200 & 344,4 & 384,2 \\
\hline
\end{tabular}

Tabl. 2

Ce qui saute aux yeux, ce sont les périodes de forte croissance des activités métalinguistiques, d'abord au milieu du 16e siècle (notamment en France et en Espagne) et surtout entre le milieu du 18e jusqu'au milieu du 19e siècle. Dans l'historiographie linguistique on a souvent souligné la coïncidence de cette première normalisation et grammaticalisation des langues vulgaires au 16e siècle avec l'expansion territoriale, voire coloniale - notamment celle de l'Espagne en Afrique du Nord et en Amérique - et la constitution des États nationaux ${ }^{4}$. L'autre facteur souvent mentionné, c'est l'établissement de l'imprimerie qui fait naître un marché pour toute sorte de publication en

4 L'unification nationale est une valeur centrale dans la légitimation de l'enseignement de la langue maternelle, notamment en France. Gadet (1999:637) montre que le but de renforcer cette unité se trouve explicitement mentionné dans les Instructions officielles des années 1920. 
langue vulgaire et qui crée ainsi la nécessité d'une orientation normative. Sur le plan de l'histoire des idées, le rôle croissant de l'écrit dans l'éducation religieuse, et donc la propagation de l'alphabétisation de la part des autorités religieuses joue également son rôle ${ }^{5}$.

\subsection{Le facteur démographique}

Sans nier l'interdépendance de la normalisation ni avec le colonialisme naissant, ni avec l'imprimerie, ni avec l'histoire de idées grammaticales, nous voudrions discuter la question de savoir dans quel degré la croissance des activités métalinguistiques coïncide avec des facteurs-clef de l'évolution socio-économique. Pour valoriser la croissance impressionnante de publications métalinguistiques, nous la mettons d'abord en relation avec la croissance démographique:

\begin{tabular}{|l|r|r|r|r|r|r|}
\hline \multicolumn{2}{|c|}{ population en 1000 } & & & & & \\
\hline & vs. 1500 & vs. 1600 & vs. 1700 & vs. 1820 & vs. 1900 & vs. 2000 \\
\hline France & 15000 & 18500 & 21471 & 31250 & 40598 & 59382 \\
\hline Espagne & 6800 & 8240 & 8770 & 12203 & 18566 & 40016 \\
\hline Portugal & 1000 & 1100 & 2000 & 3297 & 5404 & 10048 \\
\hline
\end{tabular}

Tabl. 3 (source: Maddison 2007$)^{6}$

La transformation des chiffres absolus en taux de croissance démographique permet de mieux comparer les données:

\begin{tabular}{|l|r|r|r|r|r|r|}
\hline \multicolumn{2}{|c|}{ croissance démographique en \%) } & & & & \\
\hline & $\mathbf{1 5 0 0 - 1 6 0 0}$ & $\mathbf{1 6 0 0 - 1 7 0 0}$ & $\mathbf{1 7 0 0 - 1 8 2 0}$ & $\mathbf{1 8 2 0 - 1 9 0 0}$ & $\mathbf{1 9 0 0 - 2 0 0 0}$ \\
\hline France & 18,9 & 13,8 & 31,3 & 23 & 31,6 \\
\hline Espagne & 17,4 & 6 & 28,2 & 34,3 & 53,6 \\
\hline Portugal & 9,1 & 45 & 39,3 & 39 & 46,2 \\
\hline
\end{tabular}

Ce que l'on constate d'abord, c'est le fait que la croissance des activités métalinguistiques surpasse largement celle de l'évolution démographique. Il y a tout de même une certaine interrelation. Notamment les périodes de faible croissance démographique coïncident avec celles de faible croissance des activités métalinguistiques, comme on voit p.ex. dans le cas du Portugal au 16e siècle ou en Espagne au 17e siècle. De surcroît, en comparaison avec la France et l'Espagne, la plus forte croissance démographique du Portugal au 17e siècle va de pair avec un taux de croissance «métalinguistique » relativement plus élevé que celui de la France ou de l'Espagne. Le ralentissement de la croissance démographique que l'on observe en France et en Espagne au 17e siècle semble freiner un petit peu le dynamisme 'grammatical'.

\subsection{Le facteur économique}

Pour comprendre le cadre social, dans lequel la constitution de normes linguistiques peut se dérouler, cela vaut la peine de regarder de près l'évolution de la productivité économique. La productivité est en général considérée comme l'échelle principale pour mesurer le développement économique d'une société. Elle permet de délimiter le degré de mobilité sociale, l'accès à l'éducation scolaire comme les transformations des structures professionnelles. Les estimations de Maddison (2007) sur la productivité économique par personne donnent les chiffres suivants:

\begin{tabular}{|l|r|r|r|r|r|r|}
\hline \multicolumn{2}{|c|}{ économie (PIB/pers.) } & & & & & \\
\hline & vs. 1500 & v.1600 & vs. 1700 & vs. 1820 & vs. 1900 & vs. 2000 \\
\hline France & 727 & 841 & 910 & 1135 & 2876 & 20808 \\
\hline Espagne & 661 & 853 & 853 & 1008 & 1786 & 15269 \\
\hline Portugal & 606 & 740 & 819 & 923 & 1302 & 14022 \\
\hline
\end{tabular}

Tabl. 5 (d'après Maddison 2007)

Ces estimations permettent de mesurer la croissance de la productivité, un indicateur du progrès technique et économique:

5 « Dès la fin du XVIe siècle, les catholiques d'après Trente en sont d'accord avec leurs rivaux protestants: l'alphabétisation est à vocation universelle. Telle est l'immense révolution » (Furet/Ozouf 1977a:492).

6 Les chiffres indiqués par Maddison ne peuvent être que des estimations approximatives. Néanmoins les estimations 'alternati ves' confirment les tendances générales de l'évolution démographique. 


\begin{tabular}{|l|r|r|r|r|r|r|}
\hline \multicolumn{2}{|c|}{ croissance de la productivité en \% } & & \\
\hline & & $\mathbf{1 5 0 0 - 1 6 0 0}$ & $\mathbf{1 6 0 0 - 1 7 0 0}$ & $\mathbf{1 7 0 0 - 1 8 0 0}$ & $\mathbf{1 8 2 0 - 1 9 0 0}$ & $\mathbf{1 9 0 0 - 2 0 0 0}$ \\
\hline France & 13,6 & 7,6 & 19,8 & 60,5 & 86,2 \\
\hline Espagne & 22,5 & 0 & 15,4 & 43,6 & 88,3 \\
\hline Portugal & 18,1 & 9,6 & 11,3 & 29,1 & 90,7 \\
\hline
\end{tabular}

Tabl. 6

A l'exception de la période du siglo de oro, ou l'économie espagnole est plus productive que celle de la France, on observe une hiérarchie des économies nationales, conduite par la France et suivie par l'Espagne. L'économie portugaise se trouve - par rapport au critère de la productivité - au dernier rang. L'écart entre les trois pays commence vraiment à s'accentuer au 19e siècle. La révolution industrielle se fait sentir plus nettement en France qu'en Espagne ou au Portugal.

En général on observe un parallélisme entre l'évolution de la productivité et celle des activités métalinguistiques. Notamment au 16e et au 19e siècle, on assiste à des périodes de forte croissance économique qui s'accompagnent d'une montée impressionnante de publications métalinguistiques. En revanche, la stagnation économique que connaît l'Espagne au $17 \mathrm{e}$ siècle va de pair avec une stagnation, voire même une diminution de $13 \%$ des activités métalinguistiques. Cette " croissance négative » trouve ainsi une explication dans l'histoire sociale et économique. Le rôle prétendument néfaste de l'enseignement des jésuites et des courants littéraires baroques (argument-clef des 'ilustrados' du siècle XVIII pour expliquer la crise intellectuelle du XVIIe siècle; Polzin-Haumann 2006) ne peut pas être totalement écarté.

Le progrès économique semble en tout cas encourager la publication de grammaires. Si on conjugue le développement de la productivité avec celui de la croissance démographique, cette tendance s'affiche encore plus clairement:

\begin{tabular}{|c|r|r|r|r|r|r|}
\hline \multicolumn{2}{|c|}{ croissance des économies nationales (PIB en millions } & & & \\
$\mathbf{1 9 9 0}$ International Geary-Khamis dollars) & $\mathbf{1 6 0 0}$ & $\mathbf{1 7 0 0}$ & $\mathbf{1 8 2 0}$ & $\mathbf{1 9 0 0}$ & $\mathbf{2 0 0 0}$ \\
\hline & $\mathbf{1 5 0 0}$ & 15559 & 19539 & 35468 & 116747 & 1263467 \\
\hline France & 10912 & 7029 & 7481 & 12299 & 33164 & 625116 \\
\hline Espagne & 4495 & 814 & 1638 & 3043 & 7037 & 142753 \\
\hline Portugal & 606 & &
\end{tabular}

Tabl. 7 (d'après Maddison 2007)

\begin{tabular}{|l|r|r|r|r|r|r|}
\hline \multicolumn{2}{|c|}{ croissance des économies nationales en \% } \\
\hline & & $\mathbf{1 5 0 0 - 1 6 0 0}$ & $\mathbf{1 6 0 0 - 1 7 0 0}$ & $\mathbf{1 7 0 0 - 1 8 2 0}$ & $\mathbf{1 8 2 0 - 1 9 0 0}$ & $\mathbf{1 9 0 0 - 2 0 0 0}$ \\
\hline France & & 42,6 & 25,6 & 81,5 & 229,2 & 982,2 \\
\hline Espagne & 56,4 & 6,4 & 64,4 & 169,6 & 1784,9 \\
\hline Portugal & 34,3 & 101,2 & 85,8 & 131,3 & 1928,6 \\
\hline
\end{tabular}

Tabl. 8

\subsection{La géographie sociale de l'alphabétisation et du discours normatif}

Étroitement lié aux conditions socio-économiques, le niveau de l'éducation est bien entendu un des facteurs centraux pour mesurer l'impact des activités métalinguistiques. La publication de grammaires s'intègre souvent dans des efforts pédagogiques et ne peut être dissociée de l'éducation, soit publique, soit privée. Ou, pour le dire plus clairement: les efforts normatifs - tels qu'ils peuvent être mesurés à travers la statistique des publications - ont pour condition préalable l'alphabétisation.

Reconstruire les taux d'alphabétisme se révèle pourtant être une entreprise difficile. Furet/Ozouf (1977, I:13-35) discutent les critères longtemps utilisés, comme p.ex. la signature de l'acte de mariage. Rita Marquilhas (2000:87) souligne le fait que l'utilisation de lettres ne correspond pas toujours à la maîtrise de l'écriture. Toutes les indications dont on dispose sont ainsi de nature incertaine. Malgré les incertitudes on peut à juste titre observer un long processus d'alphabétisation qui se déroule en France « entre Calvin et Jules Ferry » et sur la péninsule ibérique entre le 16e siècle et la transition démocratique à la fin du $20 \mathrm{e}$ siècle. 
Malgré toutes incertitudes, on peut révéler une coïncidence frappante entre l'évolution des activités métalinguistiques et celle des taux d'alphabétisme. Si on compare p.ex. les chiffres globaux de l'alphabétisation en France entre 1700 et 1900, on découvre la montée constante du taux d'alphabétisme, avec une certaine accélération au début du 19e siècle ${ }^{7}$. La montée spectaculaire du nombre de grammaires publiés (de 63 pour la période entre 1740 et 1760 à 523 pour la période entre 1840 et 1860) 'accompagne' ce processus de l'alphabétisation:

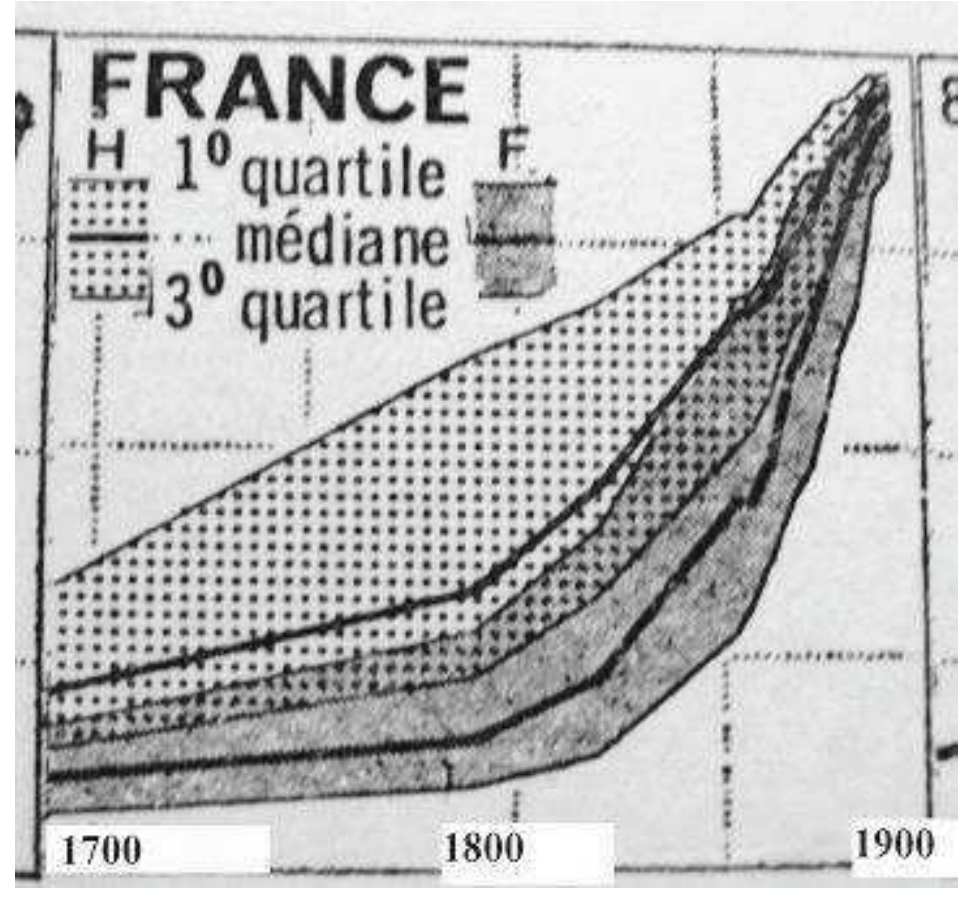

Tabl. 9 (Furet/Ozouf 1977:8)

Ce qui est intéressant à découvrir, c'est l'interdépendance entre alphabétisation et les structures économiques. Les études détaillées faites sur le déroulement de l'alphabétisation permettent de comprendre les différents facteurs qui favorisent l'apprentissage du lire-écrire. Le retard de l'Espagne et du Portugal en matière d'alphabétisation surtout au 19e siècle est assez remarquable, comme le démontre la statistique de l'alphabétisation espagnole entre 1860 et 1960. Ce retard en correspond au décalage économique entre la Péninsule ibérique et la France:

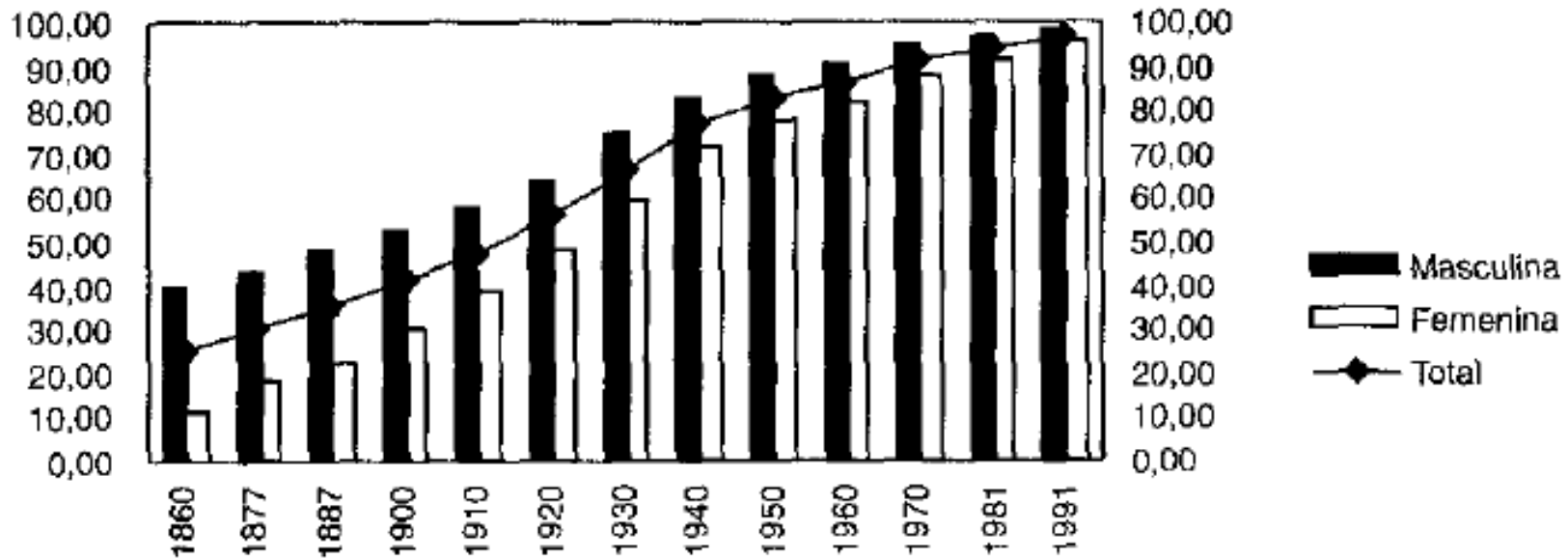

Tabl. 10 L'alphabétisation en Espagne (1860-1991), d'après Gabriel (1997:214)

En général, la condition préalable de l'alphabétisation est un minimum de prospérité:

« À quelques exceptions près (...), il existe dans la France du XVIIIe siècle un lien entre la prospérité générale d'une ré gion et son niveau d'alphabétisation. Il faut un minimum d'aisance pour que se crée cette disponibilité psychologique né-

7 Il faut prendre note des différences régionales importantes. L'alphabétisation dans les régions septentrionales se déroule à un rythme plutôt constant, tandis que la majorité des populations méridionales ne sont alphabétisées que tardivement, à partir d'environ 1820. A la fin du 19e siècle, la France est largement alphabétisée à l'exception près de la Bretagne bretonnante. 
cessaire d'une demande d'alphabétisation et pour qu'on trouve dans les fonds de fabrique, dans chaque famille de quoi as surer les revenus d'un maître d'école » (Furet/Ozouf 1977:II,117).

Dans une analyse détaillée des processus régionaux, Furet/Ozouf remarquent la différence nette entre le monde rural et les villes. Les villes - surtout les centres administratifs ou commerciaux - connaissent un taux d'alphabétisme plus élevé que les villages. L'exemple de l'ancien diocèse de Rouen (Furet/Ozouf 1977:II, 101-152) nous renseigne sur les secteurs sociaux et professionnels spécifiquement favorables à l'alphabétisation. À l'intérieur de cette vaste diocèse, les régions "tournées vers l'échange » (117) sont plus ouvertes à l'alphabétisation que d'autres régions, même celles à un niveau de prospérité comparable. Ces tendances, tirées d'une analyse assidue de la situation en Normandie du $18 \mathrm{e}$ siècle, se confirment au-delà des frontières françaises. Il y a p.ex. des parallèles avec le Portugal du 20e siècle, où l'analphabétisme était surtout un problème des régions rurales. Même à une distance de 200 ans, le fondement socio-économique de l'alphabétisation reste quasi identique ${ }^{8}$.

On peut en conclure que la présence de l'échange commerciale encourage l'alphabétisation. La mobilité géographique ainsi que les besoins pratiques du commerce favorisent donc la culture écrite. Le secteur professionnel des « marchands » connaît ainsi - à part le groupe des « notables » - le chiffre le plus élevé de personnes sachant lire et écrire (Furet/Ozouf 1977:II,144s.).

Cette réalité sociale se reflète également dans les oeuvres normatifs. À partir de la fin du $18 \mathrm{e}$ siècle, le rôle croissant du commerce figure comme argument légitimatoire des traités normatifs, comme en témoignent maintes citations, tirées surtout des préfaces de grammaires. João Pinheiro da Cunha (1791:V) souligne l'utilité de ses Conjugações Portuguezas pour « se fazerem habeis nos Empregos da Republica, e sociedade das gentes ». Les éditeurs du deuxième tirage du Secretario Portugues ( $\left.{ }^{2} 1797\right)$ de Francisco José Freire intègrent même un traité sur le commerce dans un oeuvre de nature para-grammatical:

« Esta nova Edição apparece muito mais augmentada; porque como me lembrei que neste Reino vai briosamente cami nhando (...) o amor da honrosa, e sempre brilhante occupação do commercio; para que todos vejão (...) que este genero de profissão naõ he de taõ pouco momento, como alguem pôde pensar, visto que constitue o esplendor, a força, e a riqueza das Nações, civilisa os homens, dá-lhes a conhecer a que se passa nos outros Estados, e os ennobrece, unindo-os com as jerarquias mais gradas nas Cidades, ajunto-lhe segundo Supplemento, que contem vários pontos concernentes á Theoria, e Práti ca do commercio » (Advertencia do Editor).

Dans le contexte portugais on peut même interpréter cette apologie du commerce, datant de la fin du 18e siècle, comme un jugement anti-aristocratique, vu que les professions «bourgeoises » sont ainsi valorisées à égalité avec les charges officielles, tout en soulignant leurs importance et honneur.

La même tendance s'observe en Espagne: au début du 18e siècle, les débats sur les normes linguistiques étaient principalement des débats sur la langue littéraire. Dans la deuxième moitié du 18e siècle, les guides destinés exclusivement aux écrivains font de plus en plus place à des manuels pratiques avec des fins pédagogiques. PolzinHaumann (2006:131-137) montre que les idées des «lumières » se font remarquer dans la définition du public destinataire. Les éditeurs des oeuvres normatifs évoquent de plus en plus les arguments utilitaires. La divulgation des connaissances linguistiques fait d'un côté partie du programme pédagogique des « ilustrados », d'autre côté la flambée grammaticale s'insère dans la transformation des réalités sociales ${ }^{9}$. Au $19 \mathrm{e}$ siècle finalement, on observe l'entrée du discours normatif dans les nouveau médias de masse, comme les journaux quotidiens (Osthus 2006; Lebsanft 2006).

8 Au Portugal le processus de l'alphabétisation est très en retard par rapport à la France. Rómulo de Carvalho (1999:635;711) montre qu'à la fin du 19e siècle, le taux d'analphabétisme s'élève encore à $80 \%$ : $(1878: 82,4 \% ; 1890: 79,2 \% ; 1900: 78,6 \%$; 1911: 75,1\%; 1930: 67,8\%). Ce qui est révélateur, c'est la différence entre les grandes villes et les zones rurales. Le taux d'analphabétisme était inférieur dans les villes de Lisbonne (ca. 57 \% vers 1900) et de Porto qu'à la campagne.

9 «Diese Ausweitung des von den Autoren genannten Zielpublikums zeugt von gewandelten gesellschaftlichen Bedingungen der Kommunikation. (...) [Es] wird zunehmend die Notwendigkeit guten Sprechens für einen deutlich größeren - im einzelnen durchaus unterschiedlich bestimmten - Personenkreis betont. Die rekurrente Ausdehnung der Anforderung von Sprachrichtigkeit (...) auf Männer und Frauen läßt auf eine sich wandelnde Stellung der Frau in der Gesellschaft schließen » (Polzin-Haumann 2006:136). 


\section{Conclusion}

Nous avons essayé de montrer que la constitution des normes linguistiques fait partie de l'histoire sociale des pays européens. Pour décrire les conditions sociales de la 'normativisation', nous l'avons mise en relation avec des facteursclef du développement socio-économique.

Il parait utile de distinguer l'évolution des idées normatives et l'acceptation de ces idées de la part des différents groupes sociaux. Sans adhésion sociale, aucune idée sur ce qui est la langue légitime n'a une chance de s'imposer. C'est ainsi que la fixation des langues nationales précède l'expansion des activités métalinguistiques. En France, comme en Espagne et au Portugal cette expansion sociale des normes linguistiques représente une évolution qui s'accélère surtout à partir de la deuxième moitié du 18e siècle. Même si les possibilités de quantifier les efforts normatifs à partir du chiffre des publications sont limitées, on constate que les tendances observées se conjuguent avec d'autres facteurs sociaux, comme le progrès économique, l'évolution démographique et l'histoire de l'enseignement.

Il est impossible d'établir un lien de causalité direct entre ces phénomènes. Ce que l'on peut cependant voir, c'est une forte coïncidence entre ces évolutions. Avec la croissance économique, surtout avec les progrès de productivité, l'alphabétisation des couches sociales jusqu'alors analphabètes progresse inévitablement. Les transformations sociales encouragent l'amélioration du niveau d'éducation. L'augmentation de l'échange économique - favorisée par la modernisation de l'infrastructure et la lente transformation d'une société agraire en une économie proto-industrielle contribue à valoriser la maîtrise de la langue écrite. L'adaptation des usages linguistiques aux normes établies devient ainsi un facteur important de promotion sociale. Ceci est moins le résultat d'une politique volontariste de la langue que de l'avènement de la modernité, ou - si j'ose dire - de la « révolution tranquille » des temps modernes.

Nous avons conclu que les normes linguistiques s'avèrent importantes pour la promotion sociale. En même temps, les comportements linguistiques sont soumis aux jugements méta-normatifs, c'est-à dire que l'usage linguistique devient également un facteur potentiel de discrimination sociale; une discrimination dont Pierre Bourdieu décrit les mécanismes.

C'est à l'historiographie des normes linguistiques d'inclure les aspects sociaux que nous venons d'esquisser. La coïncidence des différents facteurs économique, démographique et métalinguistique reste à prouver au niveau local. Le regard un peu global sur une évolution de 400 ans invite à se pencher sur les problèmes en détail. La construction d'un éventuel modèle décrivant l'interrelation entre le changement social et la constitution de normes linguistiques demande encore beaucoup de travail 'sur le terrain'. Le temps est en tout cas venu d'aborder une histoire intégrale de la normalisation linguistique. À côté de plusieurs aspects très importants - que nous avons abordé ces derniers jours dans nos débats - l'histoire sociale de la norme linguistique est d'un intérêt essentiel.

\section{Bibliographie}

Auroux, Silvain (ed. 1992), Histoire des idées linguistiques, II. Le développement de la grammaire occidentale, Liège/Bruxelles.

Auroux, Sylvain (1998), La raison, le langage et les normes, Paris.

Berrendonner, Alain (1982), L'éternel grammairien. Étude du discours normatif, Berne.

Bourdieu, Pierre (1983), Ce que parler veut dire. L'économie des échanges linguistiques, Paris.

Bourdieu, Pierre (2001), Langage et pouvoir symbolique, Paris.

Burke, Peter/Porter, Roy (edd. ${ }^{3}$ 1994), The social history of language, Cambridge.

Cardoso, Simão (1994), Historiografia gramatical 1500-1920, Porto.

Carvalho, Rómulo de ("32001), História do ensino em Portugal desde a fundação da nacionalidade até o fim do regime de Salazar-Caetano, Lisboa.

Catach, Nina (2001), Histoire de l'orthographe française, Paris.

Certeau, Michel de/ Julia, Dominique/Revel, Jacques (1975), Une politique de la langue. La Révolution française et les patois: L'enquête de Grégoire, Paris.

Corbeil, Jean-Claude (1983), «Éléments d'une théorie de la régulation linguistique », In: Bédard, Edith/Maurais, Jacques (edd. 1983), La norme linguistique, Québec, 281-303.

Cunha, João Pinheiro Freire da (1791), Conjugações portuguezas regulares e irregulares, Lisboa.

Desgrouais (1801), Les Gasconismes corrigés, Toulouse, 1801 [1 1766; ${ }^{2} 1768$; ND Nîmes, 1997].

Diogo, Américo António Lindeza/Silvestre, Osvaldo Manuel (1996), Rumo ao Português legítimo. Língua e Literatura (1750-1850), Braga.

Freire, Francisco Jozé ( $\left.{ }^{2} 1797\right)$, Secretario portuguez, ou methodo de escrever cartas, Lisboa.

Fries, Dagmar (1989), 'Limpia, fija y da esplendor'. La Real Academia Española ante el uso de la lengua, Madrid. 
Furet, François/Ozouf, Jacques (1977), Lire et écrire. L'alphabétisation des français de Calvin à Jules Ferry, 2 tomes, Paris.

Furet, François/Ozouf, Jacques (1977a), « Trois siècles de métissage culturel », In: Annales ESC (32/3), 488-502.

Gabriel, Narciso de (1997), «Alfabetización, semialfabetización y analfabetismo en Espana (1860-1991) », In: Revista Complutense de Educación 1, 199-231

Gadet, Françoise (1999), «La langue française au XXe siècle. I. L'émergence de l'oral », In: Chaurand, Jacques (coord.), Nouvelle Histoire de la langue française, Paris, 583-667.

Gärtner, Eberhard (1999), «Zur Problematik der Periodisierung der Geschichte der portugiesischen Sprache », In: Große, Sybille/Schönberger, Axel (edd. 1999), Dulce et decorum est philologiam colere. Festschrift für Dietrich Briesemeister zu seinem 65. Geburstag, , Berlin, I, 883-896.

Lebsanft, Franz (1997), Spanische Sprachkultur. Studien zur Bewertung und Pflege des öffentlichen Sprachgebrauchs im heutigen Spanien, Tübingen.

Niederehe, Hans-Josef (1995; 1999; 2005), Bibliografía cronológica de la lingüística, la gramática y la lexicografía del español (BICRES), Amsterdam/Philadelphia.

Maddison, Angus (2007) World Population, GDP and Per Capita GDP, 1-2003 AD, Groningen [http://www.ggdc.net/maddison/Historical_Statistics/horizontal-file_03-2007.xls; 30.8.2007]

Marquilhas, Rita (2000), A Faculdade das Letras - Leitura e escrita em Portugal no séc. XVIII, Lisboa.

Mattheier, Klaus J. (1999), « Sprachhistoriker als Soziologen. Über sprachwissenschaftliche Versuche zur Strukturierung sozialer Gemeinschaften », In: Gardt, Andreas/Haß-Zumkehr, Ulrike/Roelcke, Thorsten (edd. 1999), Sprachgeschichte als Kulturgeschichte, Berlin/New York, 11-18.

Osthus, Dietmar (2006), « Laienlinguistik und Sprachchroniken. Französisch/Okzitanisch », In: Ernst, Gerhard/ Glessgen, Martin-Dietrich/ Schmitt, Christian/ Schweickard, Wolfgang (edd.), Romanische Sprachgeschichte - Histoire des langues romanes. Ein internationales Handbuch zur Geschichte der romanischen Sprachen und ihrer Erforschung, Berlin. [= Handbücher zur Sprach- und Kommunikationswissenschaft 23.2], 1533-1546.

Polzin-Haumann, Claudia (2006), Sprachreflexion und Sprachbewußtsein. Beitrag zu einer integrativen Sprachgeschichte des Spanischen im 18. Jahrhundert, Frankfurt a. M. e.a.

Settekorn, Wolfgang (1988), Sprachnorm und Sprachnormierung in Frankreich. Einführung in die begrifflichen, historischen und materiellen Grundlagen, Tübingen.

Settekorn, Wolfgang (1988b), « Normalisation et différenciation: Réflexions sur la naissance et les effets de la langue imprimée en France », in: Kremer, Dieter/Schlieben-Lange, Brigitte (edd.), Actes du XVIIIe Congres International de Linguistique et de Philologie Romanes, V: Section IV: Linguistique pragmatique et linguistique sociolinguistique, Tübingen, 55-67.

Spillner, Bernd (2004), «Les Gasconismes corrigés: Die Bedeutung korrektiver Traktate für Sprachkontakt und Sprachvarietät in Frankreich », In: Gil, Alberto/Osthus, Dietmar/Polzin-Haumann, Claudia (edd. 2004), Romanische Sprachwissenschaft. Zeugnisse für Einheit und Vielfalt eines Faches. Festschrift für Christian Schmitt anläßlich seines 60. Geburtstages, Frankfurt a. M. e.a., I, 107-122.

Stengel, Edmund (1890 [ed. Niederehe, Hans Josef 1976]), Chronologisches Verzeichnis französischer Grammatiken, Tübingen.

Thielemann, Werner (2004), « Soziales Umfeld - sprachliches Prestige. Zu Uso und Pureza in Portugal zwischen Restauração und Luzes ", in: Gil, Alberto/Osthus, Dietmar/Polzin-Haumann, Claudia (edd. 2004), Romanische Sprachwissenschaft. Zeugnisse für Einheit und Vielfalt eines Faches. Festschrift für Christian Schmitt anläßlich seines 60. Geburtstages, Frankfurt a. M. e.a., I, 123-144.

Thompson, John B. (2001 [1991]), « Préface », in: Bourdieu, Pierre, Langage et pouvoir symbolique, Paris, 7-51. 